\title{
Zeta-functions for germs of meromorphic functions and Newton diagrams
}

\author{
S.M. Gusein-Zade \\ I. Luengo \\ A. Melle-Hernández *
}

\begin{abstract}
For a germ of a meromorphic function $f=\frac{P}{Q}$, we offer notions of the monodromy operators at zero and at infinity. If the holomorphic functions $P$ and $Q$ are non-degenerated with respect to their Newton diagrams, we give an analogue of the formula of Varchenko for the zeta-functions of these monodromy operators.
\end{abstract}

\section{Germs of meromorphic functions}

A polynomial $f$ of $(n+1)$ complex variables of degree $d$ determines a meromorphic function $f$ on $\mathbb{C P}^{n+1}$. If one wants to understand the behaviour of $f$ at infinity, it is natural to analize germs of the meromorphic function $f$ at points from the infinite hyperplane $\mathbb{C P}_{\infty}^{n} \subset \mathbb{C P}^{n+1}$. In local analytic coordinates $z_{0}, z_{1}, \ldots, z_{n}$, centred at a point $p \in \mathbb{C P}_{\infty}^{n}$ such that the infinite hyperplane $\mathbb{C P}_{\infty}^{n}$ is given by the equation $\left\{z_{0}=0\right\}$, the germ of the function $f$ has the form $f=\frac{P\left(z_{0}, \ldots, z_{n}\right)}{z_{0}^{d}}$. Let us consider germs of meromorphic functions of a general form.

DEFINITION 1 A germ of a meromorphic function on $\left(\mathbb{C}^{n+1}, 0\right)$ is a fraction $f=\frac{P}{Q}$, where $P$ and $Q$ are germs of holomorphic functions $\left(\mathbb{C}^{n+1}, 0\right) \rightarrow(\mathbb{C}, 0)$. Two germs of meromorphic functions $f=\frac{P}{Q}$ and $f^{\prime}=\frac{P^{\prime}}{Q^{\prime}}$ are equal if there exists a germ of a holomorphic function $U:\left(\mathbb{C}^{n+1}, 0\right) \rightarrow \mathbb{C}$ such that $U(0) \neq 0, P^{\prime}=U \cdot P$ and $Q^{\prime}=U \cdot Q$.

REMARKS. (1) For our convenience here we do not consider functions of the type $\frac{1}{Q(z)}$ or $\frac{P(z)}{1}$.

(2) According to the definition $\frac{x}{y} \neq \frac{x^{2}}{x y}$, but $\frac{x}{y}=\frac{x \exp (x)}{y \exp (x)}$.

Recently V.I. Arnold had obtained classifications of simple germs of meromorphic functions for certain equivalence relations.

In what follows we shall consistently use resolutions of germs of meromorphic functions.

${ }^{*}$ First author is partially supported by RFBR-95-01-01122a and INTAS-4373. Last two authors are partially supported by CAICYT PB94-291. 
DEFINITION 2 A resolution of the germ $f$ is a modification of $\left(\mathbb{C}^{n+1}, 0\right)$ (i.e. a proper analytic map $\pi: \mathcal{X} \rightarrow \mathcal{U}$ of a smooth analytic manifold $\mathcal{X}$ onto a neighbourhood $\mathcal{U}$ of the origin in $\mathbb{C}^{n+1}$, which is an isomorphism outside of a proper analytic subspace in $\mathcal{U}$ ) such that the total transform $\pi^{-1}(H)$ of the hypersurface $H=\{P=0\} \cup\{Q=0\}$ is a normal crossing divisor at each point of $\mathcal{X}$.

The fact that the preimage $\pi^{-1}(H)$ is a divisor with normal crossings implies that in a neighbourhood of any point of it, there exists a local system of coordinates $y_{0}, y_{1}, \ldots, y_{n}$ such that the liftings $\tilde{P}=P \circ \pi$ and $\tilde{Q}=Q \circ \pi$ of the functions $P$ and $Q$ to the space $\mathcal{X}$ of the modification are equal to $u y_{0}^{k_{0}} y_{1}^{k_{1}} \cdots y_{n}^{k_{n}}$ and $v y_{0}^{l_{0}} y_{1}^{l_{1}} \cdots y_{n}^{l_{n}}$ respectively, where $u(0) \neq 0$ and $v(0) \neq 0$.

Let $B_{\varepsilon}$ be the closed ball of radius $\varepsilon$ with the centre at the origin in $\mathbb{C}^{n+1}$ and $\varepsilon$ be small enough such that (representatives of) the functions $P$ and $Q$ are defined in $B_{\varepsilon}$ and for any positive $\varepsilon^{\prime}<\varepsilon$ the sphere $S_{\varepsilon^{\prime}}=\partial B_{\varepsilon^{\prime}}$ intersects the analytic spaces $\{P=0\},\{Q=0\}$ and $\{P=Q=0\}$ transversally (in the stratified sense). We choose $\delta$ small enough and take the ball $B_{\delta} \subset \mathbb{C}^{2}$ of radius $\delta$ centred at the origin.

DEFINITION 3 Let $c \in \mathbb{C}$ be such that $\|c\|$ is small enough, the 0-Milnor fibre $\mathcal{M}_{f}^{0}$ of the germ $f$ is the set

$$
\mathcal{M}_{f}^{0}=\left\{z \in B_{\varepsilon}:(P(z), Q(z)) \in B_{\delta} \subset \mathbb{C}^{2}, f(z)=\frac{P(z)}{Q(z)}=c\right\}
$$

In the same way, for $c \in \mathbb{C}$ such that $\|c\|$ is large enough, the $\infty$-Milnor fibre $\mathcal{M}_{f}^{\infty}$ of the germ $f$ is the set

$$
\mathcal{M}_{f}^{\infty}=\left\{z \in B_{\varepsilon}:(P(z), Q(z)) \in B_{\delta} \subset \mathbb{C}^{2}, f(z)=\frac{P(z)}{Q(z)}=c\right\}
$$

LEMMA 1 The notion of the 0- (respectively of the $\infty$-) Milnor fibre is well defined, i.e. for $\|c\|$ small enough: $\|c\| \ll \delta \ll \varepsilon$ (respectively for $\|c\|$ large enough: $\|c\|^{-1} \ll \delta \ll \varepsilon$ ) the differentiable type of $\mathcal{M}_{f}^{0}$ (respectively of $\mathcal{M}_{f}^{\infty}$ ) does not depend on $\varepsilon, \delta$ and $c$.

Proof. Let $\pi: \mathcal{X} \rightarrow \mathcal{U}$ be a resolution of the germ $f$ which is an isomorphism outside the hypersurface $H=\{P=0\} \cup\{Q=0\}$. Let $r: \mathbb{C}^{n+1} \rightarrow \mathbb{R}$ be the function $r(z)=\|z\|^{2}$, let $\tilde{r}=r \circ \pi: \mathcal{X} \rightarrow \mathbb{R}$ be the lifting of $r$ to the space $\mathcal{X}$ of the resolution. For $\varepsilon$ small enough, the hypersurface $\tilde{S}_{\varepsilon}=\left\{\tilde{r}=\varepsilon^{2}\right\}$ (the preimage of the sphere $S_{\varepsilon} \subset \mathbb{C}^{n+1}$ ) is transversal to all components of the total transform $\pi^{-1}(H)$. At each point of $\pi^{-1}(H)$ in a local coordinate system one has $P \circ \pi=u y_{0}^{k_{0}} \cdots y_{n}^{k_{n}}, Q \circ \pi=v y_{0}^{l_{0}} \cdots y_{n}^{l_{n}}$ with $u(0) \neq 0$ and $v(0) \neq 0$. Thus $f \circ \pi=w y_{0}^{m_{0}} \cdots y_{n}^{m_{n}}$ with $w(0) \neq 0$. The real hypersurface $\tilde{S}_{\varepsilon}$ is transversal to all coordinate subspaces (of different dimensions). It is not difficult to show that this implies transversality of $\tilde{S}_{\varepsilon}$ to the (complex) hypersurfaces $\left\{w y_{0}^{m_{0}} \cdots y_{n}^{m_{n}}=c\right\}$ for $\|c\|$ small enough and for $\|c\|$ large enough. Now the proof follows from the standard arguments. 
REMARKS. (1) The definition means that $\mathcal{M}_{f}^{0}$ or $\mathcal{M}_{f}^{\infty}$ is equal to

$$
\left\{z \in B_{\varepsilon}:(P(z), Q(z)) \in B_{\delta} \subset \mathbb{C}^{2}, P(z)=c Q(z), \quad P(z) \neq 0\right\}
$$

and thus the Milnor fibres of the functions $\frac{P}{Q}$ and $\frac{R P}{R Q}$ with $R(0)=0$ are, generally speaking, different.

(2) For $f=\frac{P}{Q}$, let $f^{-1}=\frac{Q}{P}$. It is not difficult to understand that $\mathcal{M}_{f^{-1}}^{0}=\mathcal{M}_{f}^{\infty}$ and $\mathcal{M}_{f^{-1}}^{\infty}=\mathcal{M}_{f}^{0}$. Just the same properties hold for the monodromy transformations and for the zeta-functions discussed bellow.

(3) It is possible (and sometimes more convenient) to define the Milnor fibres as follows:

$$
\mathcal{M}_{f}^{0}=\left\{z \in B_{\varepsilon}:\|Q(z)\| \leq \delta, P(z)=c Q(z) \neq 0\right\}
$$

with $\|c\| \ll \delta \ll \varepsilon$, and

$$
\mathcal{M}_{f}^{\infty}=\left\{z \in B_{\varepsilon}:\|P(z)\| \leq \delta, P(z)=c Q(z) \neq 0\right\}
$$

with $\|c\|^{-1} \ll \delta \ll \varepsilon$.

The meromorphic function $f$ determines a map from $B_{\varepsilon} \backslash\{P=Q=0\}$ to the projective line $\mathbb{C P}^{1}(z \mapsto(P(z): Q(z)))$, which also will be denoted by $f$. Lemma 1 implies that this map is a locally trivial fibration in punctured neighbourhoods of the points $0=(0: 1)$ and $\infty=(1: 0)$ of $\mathbb{C P}^{1}$.

DEFINITION 4 The 0-monodromy transformation $h_{f}^{0}$ (respectively the $\infty$-monodromy transformation $h_{f}^{\infty}$ ) of the germ $f$ is the monodromy transformation of the fibration $f$ over the loop $c \cdot \exp (2 \pi i t), t \in[0,1]$, with $\|c\|$ small enough (respectively large enough).

The 0 - or $\infty$ - monodromy operator is the action of the corresponding monodromy transformation in a homology group of the Milnor fibre. We are interested to apply the results for meromorphic functions to the problem of calculating the zeta-function of a polynomial at infinity. Thus we shall consider the zeta-functions $\zeta_{f}^{0}(t)$ and $\zeta_{f}^{\infty}(t)$ of the corresponding monodromy transformations:

$$
\zeta_{f}^{\bullet}=\prod_{q \geq 0}\left\{\operatorname{det}\left[i d-\left.t h_{f *}^{\bullet}\right|_{H_{q}\left(\mathcal{M}_{f}^{\bullet} ; \mathbb{C}\right)}\right]\right\}^{(-1)^{q}}
$$

$(\bullet=0$ or $\infty)$. This definition coincides with that in $[2]$ and differs by minus sign in the exponent from that in [1].

\section{Resolution of singularities and the formula of A'Campo for germs of meromorphic functions}

Let $f=\frac{P}{Q}$ be a germ of a meromorphic function on $\left(\mathbb{C}^{n+1}, 0\right)$ and let $\pi: \mathcal{X} \rightarrow \mathcal{U}$ be a resolution of the germ $f$. The preimage $\mathcal{D}=\pi^{-1}(0)$ of the origin of $\mathbb{C}^{n+1}$, is a 
normal crossing divisor. Let $S_{k, l}$ be the set of points of $\mathcal{D}$ in a neighbourhood of which the functions $P \circ \pi$ and $Q \circ \pi$ in some local coordinates have the form $u y_{0}^{k}$ and $v y_{0}^{l}$ respectively $(u(0) \neq 0, v(0) \neq 0)$. A slight modification of the arguments of A'Campo ([1]) permits to obtain the following version of his formula for the zeta-function of the monodromy of a meromorphic function.

THEOREM 1 Let the resolution $\pi: \mathcal{X} \rightarrow \mathcal{U}$ be an isomorphism outside the hypersurface $H=\{P=0\} \cup\{Q=0\}$. Then

$$
\begin{aligned}
\zeta_{f}^{0}(t) & =\prod_{k>l}\left(1-t^{k-l}\right)^{\chi\left(S_{k, l}\right)}, \\
\zeta_{f}^{\infty}(t) & =\prod_{k<l}\left(1-t^{l-k}\right)^{\chi\left(S_{k, l}\right)} .
\end{aligned}
$$

REMARK. A resolution $\pi$ of the germ $f^{\prime}=\frac{R P}{R Q}$ is at the same time a resolution of the germ $f=\frac{P}{Q}$. Moreover the multiplicities of any component $C$ of the exceptional divisor in the zero divisors of the liftings $(R P) \circ \pi$ and $(R Q) \circ \pi$ of the germs $R P$ and $R Q$ are obtained from those of the germs $P$ and $Q$ by adding one and the same integer, the multiplicity $m=m(C)$ of $R$. Nevertheless the meromorphic functions $f$ and $f^{\prime}$ can have different zeta-functions. The reason why formulae in the previous theorem give different results for $f$ and $f^{\prime}$ consists in the fact that if an open part of the component $C$ lies in $S_{k, l}(f)$ then, generally speaking, its part which lies in $S_{k+m, l+m}\left(f^{\prime}\right)$ is smaller.

\section{Zeta-functions of meromorphic functiuons via partial resolutions}

Let $f=\frac{P}{Q}$ be a germ of a meromorphic function on $\left(\mathbb{C}^{n+1}, 0\right)$ and let $\pi:(\mathcal{X}, \mathcal{D}) \rightarrow$ $\left(\mathbb{C}^{n+1}, 0\right)$ be an arbitrary modification of $\left(\mathbb{C}^{n+1}, 0\right)$, which is an isomorphism outside the hypersurface $H=\{P=0\} \cup\{Q=0\}$ (i.e. $\pi$ is not necessarily a resolution). Let $\varphi=f \circ \pi$ be the lifting of $f$ to the space of the modification, i.e. the meromorphic function $\frac{P \circ \pi}{Q \circ \pi}$. For a point $x \in \pi^{-1}(H)$, let $\zeta_{\varphi, x}^{0}(t)$ and $\zeta_{\varphi, x}^{\infty}(t)$ be the zeta-functions of the 0 - and $\infty$ monodromies of the germ of the function $\varphi$ at $x$. Let $\mathcal{S}=\{\Xi\}$ be a prestratification of $\mathcal{D}=\pi^{-1}(0)$ (that is a partitioning into semi-analytic subspaces without any regularity conditions) such that, for each stratum $\Xi$ of $\mathcal{S}$, the zeta-functions $\zeta_{\varphi, x}^{0}(t)$ and $\zeta_{\varphi, x}^{\infty}(t)$ do not depend on $x$, for $x \in \Xi$. We denote this zeta-functions by $\zeta_{\Xi}^{0}$ and by $\zeta_{\Xi}^{\infty}$ respectively. The same arguments which were used in [4] imply

THEOREM 2 For $\bullet=0$ or $\infty$,

$$
\zeta_{f}^{\bullet}(t)=\prod_{\Xi \in \mathcal{S}}\left[\zeta_{\Xi}^{\bullet}(t)\right]^{\chi(\Xi)} .
$$




\section{Zeta-functions via Newton diagrams}

For a germ $R=\sum a_{k} x^{k}:\left(\mathbb{C}^{n+1}, 0\right) \rightarrow(\mathbb{C}, 0)$ of a holomorphic function $\left(k=\left(k_{0}, k_{1}, \ldots, k_{n}\right)\right.$, $\left.x^{k}=x_{0}^{k_{0}} x_{1}^{k_{1}} \cdots x_{n}^{k_{n}}\right)$, its Newton diagram $\Gamma=\Gamma(R)$ is the union of the compact faces of the polytope $\Gamma_{+}=\Gamma_{+}(R)=$ convex hull of $\bigcup_{k: a_{k} \neq 0}\left(k+\mathbb{R}_{+}^{n+1}\right) \subset \mathbb{R}_{+}^{n+1}$.

Let $f=\frac{P}{Q}$ be a germ of a meromorphic function on $\left(\mathbb{C}^{n+1}, 0\right)$ and let $\Gamma_{1}=\Gamma(P)$ and $\Gamma_{2}=\Gamma(Q)$ be the Newton diagrams of $P$ and $Q$. We call the pair $\Lambda=\left(\Gamma_{1}, \Gamma_{2}\right)$ of Newton diagrams $\Gamma_{1}$ and $\Gamma_{2}$ the Newton pair of $f$. We say that the germ of the meromorphic function $f$ is non-degenerated with respect to its Newton pair $\Lambda=\left(\Gamma_{1}, \Gamma_{2}\right)$ if the pair of germs $(P, Q)$ is non-degenerated with respect to the pair $\Lambda=\left(\Gamma_{1}, \Gamma_{2}\right)$ in the sense of [7] (which is an adaptation for germs of complete intersections of the definition of A.G. Khovanskii, [5]).

Let us define zeta-functions $\zeta_{\Lambda}^{0}(t)$ and $\zeta_{\Lambda}^{\infty}(t)$ for a Newton pair $\Lambda=\left(\Gamma_{1}, \Gamma_{2}\right)$. Let $1 \leq l \leq n+1$ and let $\mathcal{I}$ be a subset of $\{0,1, \ldots, n\}$ with the number of elements $\# \mathcal{I}$ equal to $l$. Let $L_{\mathcal{I}}$ be the coordinate subspace $L_{\mathcal{I}}=\left\{k \in \mathbb{R}^{n+1}: k_{i}=0\right.$ for $\left.i \notin \mathcal{I}\right\}$ and $\Gamma_{i, \mathcal{I}}=\Gamma_{i} \cap L_{\mathcal{I}} \subset L_{\mathcal{I}}$. Let $L_{\mathcal{I}}^{*}$ be the dual of $L_{\mathcal{I}}$ and $L_{\mathcal{I}+}^{*}$ the positive orthant of it ( the set of covectors which have positive values on $L_{\mathcal{I} \geq 0}=\left\{k \in L_{\mathcal{I}}: k_{i} \geq 0\right.$ for $\left.\left.i \in \mathcal{I}\right\}\right)$. For a primitive integer covector $a \in\left(\mathbb{R}^{*}\right)_{+}^{n+1}$, let $m(a, \Gamma)=\min _{x \in \Gamma}(a, x)$ and let $\Delta(a, \Gamma)=$ $\{x \in \Gamma:(a, x)=m(a, \Gamma)\}$. We denote by $m_{\mathcal{I}}$ and $\Delta_{\mathcal{I}}$ the corresponding objects for the diagram $\Gamma_{\mathcal{I}}$ and a primitive integer covector $a \in L_{\mathcal{I}+}^{*}$. Let $E_{\mathcal{I}}$ be the set of primitive integer covectors $a \in L_{\mathcal{I}}^{*}$ such that $\operatorname{dim}\left(\Delta\left(a, \Gamma_{1}\right)+\Delta\left(a, \Gamma_{2}\right)\right)=l-1$ (the Minkowski sum $\Delta_{1}+\Delta_{2}$ of two polytopes $\Delta_{1}$ and $\Delta_{2}$ is the polytope $\left.\left\{x=x_{1}+x_{2}: x_{1} \in \Delta_{1}, \quad x_{2} \in \Delta_{2}\right\}\right)$. There exists only a finite number of such covectors. For $a \in E_{\mathcal{I}}$, let $\Delta_{1}=\Delta\left(a, \Gamma_{1}\right), \Delta_{2}=$ $\Delta\left(a, \Gamma_{2}\right)$ and

$$
V_{a}=\sum_{s=0}^{l-1} V_{l-1}(\underbrace{\Delta_{1}, \ldots, \Delta_{1}}_{s \text { terms }}, \underbrace{\Delta_{2}, \ldots, \Delta_{2}}_{l-1-s \text { terms }}),
$$

where the definition of the (Minkowski) mixed volumeV $\left(\Delta_{1}, \ldots, \Delta_{m}\right)$ can be found e.g. in [3] or [7]; $(l-1)$-dimensional volume in a rational $(l-1)$-dimensional affine subspace of $L_{\mathcal{I}}$ has to be normalized in such a way that the volume of the unit cube spanned by any integer basis of the corresponding linear subspace is equal to 1 . Let us recall that $V_{m}(\underbrace{\Delta, \ldots, \Delta}_{m \text { terms }})$ is simply the $m$-dimensional volume of $\Delta$. We have to assume that $V_{0}($ nothing $)=1$, (this is necessary to define $V_{a}$ for $\left.l=1\right)$. Let:

$$
\begin{aligned}
\zeta_{\mathcal{I}}^{0}(t) & =\prod_{a \in E_{\mathcal{I}}: m\left(a, \Gamma_{1}\right)>m\left(a, \Gamma_{2}\right)}\left(1-t^{m\left(a, \Gamma_{1}\right)-m\left(a, \Gamma_{2}\right)}\right)^{(l-1) ! V_{a}}, \\
\zeta_{\mathcal{I}}^{\infty}(t) & =\prod_{a \in E_{\mathcal{I}}: m\left(a, \Gamma_{1}\right)<m\left(a, \Gamma_{2}\right)}\left(1-t^{m\left(a, \Gamma_{2}\right)-m\left(a, \Gamma_{1}\right)}\right)^{(l-1) ! V_{a}} \\
\zeta_{l}^{\bullet}(t) & =\prod_{\mathcal{I}: \#(\mathcal{I})=l} \zeta_{\mathcal{I}}^{\bullet}(t), \\
\zeta_{\Lambda}^{\bullet}(t) & =\prod_{l=1}^{n+1}\left(\zeta_{l}^{\bullet}(t)\right)^{(-1)^{l-1}}
\end{aligned}
$$


where $\bullet=0$ or $\infty$.

THEOREM 3 Let $f=\frac{P}{Q}$ be a germ of a meromorphic function on $\left(\mathbb{C}^{n+1}, 0\right)$ non-degenerated with respect to its Newton pair $\Lambda=\left(\Gamma_{1}, \Gamma_{2}\right)$. Then

$$
\zeta_{f}^{0}(t)=\zeta_{\Lambda}^{0}(t) \quad \text { and } \quad \zeta_{f}^{\infty}(t)=\zeta_{\Lambda}^{\infty}(t)
$$

Proof. Let $\Sigma$ be an unimodular simplicial subdivision of $\mathbb{R}_{\geq 0}^{n+1}$ which corresponds to the pair $\left(\Gamma_{1}, \Gamma_{2}\right)$ of Newton diagrams in the sense of [7] Section 4. This subdivision is consistent with each of the Newton diagrams $\Gamma_{1}$ and $\Gamma_{2}$ in the sense of [8].

Let $\pi:(\mathcal{X}, \mathcal{D}) \rightarrow\left(\mathbb{C}^{n+1}, 0\right)$ be the toroidal modification map corresponding to $\Sigma$. Since the pair $(P, Q)$ is non-degenerated with respect to $\left(\Gamma_{1}, \Gamma_{2}\right), \pi$ is a resolution of the germ $f=\frac{P}{Q}$ (see [7]). We have the sets $S_{k, l}=S_{k}(P) \cap S_{l}(Q)$. The description of $S_{k}(P)$ (and of $\left.S_{l}(Q)\right)$ can be found in [8], Section 7. Each of them consists of open parts of certain complex tori of some dimensions.

Tori of dimension $n$ correspond to one-dimensional cone of $\Sigma$ which are positive (i.e., lie in $\left(\mathbb{R}^{*}\right)_{+}^{n+1}$ ). The multiplicity of $P \circ \pi$ (respectively of $Q \circ \pi$ ) at such a torus is equal to $m\left(a, \Gamma_{1}\right)$, (respectively to $m\left(a, \Gamma_{2}\right)$ ) for the primitive integer covector $a$ which spans the corresponding cone.

Tori of dimension $(l-1)$ correspond to positive simplicial $(n+2-l)$-dimensional cones of $\Sigma$ which have a cone of the form

$$
\mathfrak{S}=\left\{a \in\left(\mathbb{R}^{*}\right)_{\geq 0}^{n+1}: a_{j}>0 \text { for } j \notin \mathcal{I}, \quad a_{j}=0 \text { for } j \in \mathcal{I}\right\}
$$

with $\#(\mathcal{I})=l$ (these cones are elements of $\Sigma$ ) as its face. Moreover these cones correspond to one-dimensional cones of a partitioning of $L_{\mathcal{I}}$ which is consistent with the Newton diagrams $\Gamma_{i, \mathcal{I}}=\Gamma_{i} \cap L_{\mathcal{I}} \subset L_{\mathcal{I}}$. The multiplicities of $P \circ \pi$ and $Q \circ \pi$ at such a torus again are equal to $m_{\mathcal{I}}\left(a, \Gamma_{1, \mathcal{I}}\right)$ and $m_{\mathcal{I}}\left(a, \Gamma_{2, \mathcal{I}}\right)$ for the primitive integer covector $a$ from the corresponding one-dimensional cone.

In order to apply Theorem 1 we have to calculate the Euler characteristic of the corresponding part of an $l$-1-dimensional torus $T$ : the complement to the intersection with the strict transform of the hypersurface $H=\{P=0\} \cup\{Q=0\}$. Let $A$ (respectively $B$ ) the intersection of the torus $T$ with the strict transform of the hypersurface $\{P=0\}$ (respectively of $\{Q=0\})$, let $\Delta_{i}:=\Delta\left(a, \Gamma_{i, \mathcal{I}}\right)$. From the results of Khovanskii ([6]) it follows that the Euler characteristic of $A$ (respectively of $B$ ) is equal to $(-1)^{l}(l-1) ! V_{l-1}(\underbrace{\Delta_{1}, \ldots, \Delta_{1}}_{l-1 \text { terms }})$

(respectively to $(-1)^{l}(l-1) ! V_{l-1}(\underbrace{\Delta_{2}, \ldots, \Delta_{2}}_{l-1 \text { terms }})$ ), the Euler charecteristic of $A \cap B$ is equal to

$$
(-1)^{l-1}(l-1) !\left[V_{l-1}(\underbrace{\Delta_{1}, \ldots, \Delta_{1}}_{l-2 \text { terms }}, \Delta_{2})+V_{l-1}(\underbrace{\Delta_{1}, \ldots, \Delta_{1}}_{l-3 \text { terms }}, \Delta_{2}, \Delta_{2})+\ldots+V_{l-1}(\Delta_{1}, \underbrace{\Delta_{2}, \ldots, \Delta_{2}}_{l-2 \text { terms }})\right] .
$$


Thus the Euler characteristic of the complement of $A \cup B$ in the torus $T$ is equal to

$$
\begin{gathered}
\chi(T)-\chi(A)-\chi(B)+\chi(A \cap B)= \\
=(-1)^{l-1}(l-1) !\left[V_{l-1}(\underbrace{\Delta_{1}, \ldots, \Delta_{1}}_{l-1 \text { terms }})+V_{l-1}(\underbrace{\Delta_{1}, \ldots, \Delta_{1}}_{l-2 \text { terms }}, \Delta_{2})+\ldots+V_{l-1}(\underbrace{\Delta_{2}, \ldots, \Delta_{2}}_{l-1 \text { terms }})\right],
\end{gathered}
$$

which implies the statement.

\section{The Varchenko type formula for $f=\frac{P}{z_{0}^{d}}$}

As we have mentioned at the beginning, in order to study the behaviour of polynomials at infinity, germs of meromorphic functions of the form $\frac{P\left(z_{0}, z_{1}, \ldots, z_{n}\right)}{z_{0}^{d}}$ have to be of interest. In this case the formulae for the zeta-functions $\zeta_{\Lambda}^{0}(t)$ and $\zeta_{\Lambda}^{\infty}(t)$ are considerably reduced. Thus let us reformulate the definition of these zeta-functions for the case when the Newton diagram $\Gamma_{2}$ consists of one point $(d, 0, \ldots, 0)$ (in terms of the Newton diagram $\Gamma:=\Gamma_{1}$ of $\left.P\right)$. The description is as follows.

Let $1 \leq l \leq n+1$ and let $\mathcal{I}$ be a subset of $\{1, \ldots, n\}$ with the number of elements $\# \mathcal{I}$ equal to $l-1$. Let $\gamma_{1}^{\mathcal{I}}, \ldots, \gamma_{j(\mathcal{I})}^{\mathcal{I}}$ be all $(l-1)$-dimensional faces of $\Gamma_{\mathcal{I} \cup\{0\}}$ and $a_{\mathcal{I}, 1}, \ldots, a_{\mathcal{I}, j(\mathcal{I})}$ the corresponding primitive covectors (normal to $\left.\gamma_{1}^{\mathcal{I}}, \ldots, \gamma_{j(\mathcal{I})}^{\mathcal{I}}\right), a_{\mathcal{I}, s}^{0}$ is the 0 th coordinate of $a_{\mathcal{I}, s}$, let $m_{s}(\mathcal{I})=\left(a_{\mathcal{I}, s}, k\right)$ for $k \in \gamma_{s}^{\mathcal{I}}$. Then

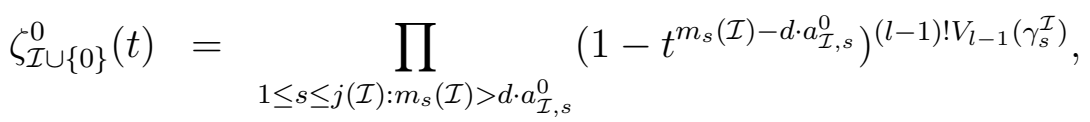

$$
\begin{aligned}
& \zeta_{\mathcal{I} \cup\{0\}}^{\infty}(t)=\prod_{1 \leq s \leq j(\mathcal{I}): m_{s}(\mathcal{I})<d \cdot a_{\mathcal{I}, s}^{0}}\left(1-t^{d \cdot a_{\mathcal{I}, s}^{0}-m_{s}(\mathcal{I})}\right)^{(l-1) ! V_{l-1}\left(\gamma_{s}^{\mathcal{I}}\right)}, \\
& \zeta_{l}^{\bullet}(t)=\prod_{\mathcal{I} \subset\{1, \ldots, n\}: \# \mathcal{I}=l-1} \zeta_{\mathcal{I} \cup\{0\}}(t), \\
& \zeta_{\Lambda}^{\bullet}(t)=\prod_{l=1}^{n+1}\left(\zeta_{l}^{\bullet}(t)\right)^{(-1)^{l-1}}
\end{aligned}
$$

$\left(\bullet=0\right.$ or $\infty$ ) where $V_{l-1}\left(\gamma_{s}^{\mathcal{I}}\right)$ is the (usual) $(l-1)$-dimensional volume of the face $\gamma_{s}^{\mathcal{I}}$ (in the hyperplane spanned by it in $\left.L_{\mathcal{I} \cup\{0\}}\right)$.

\section{$6 \quad$ Examples}

Example 1. Let $f=\frac{x^{3}-x y}{y}$. The Milnor fibre $\mathcal{M}_{f}^{0}$ (repectively $\left.\mathcal{M}_{f}^{\infty}\right)$ is $\{(x, y):\|(x, y)\|<$ $\left.\varepsilon,\left(x^{3}-x y, y\right) \in B_{\delta}, x^{3}-x y=c y\right\} \backslash\{(0,0)\}$, where $\|c\|$ is small (repectively large). From the equation $x^{3}-x y=c y$ one has $y=\frac{x^{3}}{x+c}$ and thus $\mathcal{M}_{f}^{0}$ is diffeomorphic to the disk $\mathcal{D}$ in the $x$-plane with two points removed: $-c$ and the origin. In the same way $\mathcal{M}_{f}^{\infty}$ is 
diffeomorphic to the punctured disk $\mathcal{D}^{*}$. It is not difficult to understand that the action of the monodromy transformation in the homology groups is trivial in both cases. Thus

$$
\zeta_{f}^{0}(t)=(1-t)^{-1} \quad \text { and } \quad \zeta_{f}^{\infty}(t)=1 .
$$

Now let us calculate these zeta functions from their Newton diagrams, Fig 1.

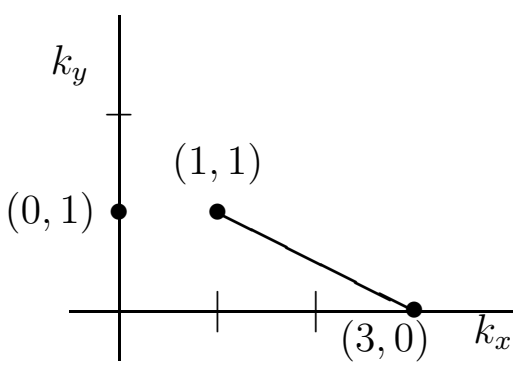

Figure 1.

We have $\zeta_{1}^{\bullet}(t)=1$ since each coordinate axis intersects only one Newton diagram. There is only one linear function (namely $\left.a=k_{x}+2 k_{y}\right)$ such that $\operatorname{dim} \Delta\left(a, \Gamma_{1}\right)=1$. The one-dimesional volume $V_{1}\left(\Delta\left(a, \Gamma_{1}\right)\right)$ of $\Delta\left(a, \Gamma_{1}\right)$ is equal to 1 and $V_{1}\left(\Delta\left(a, \Gamma_{2}\right)\right)=0$. We have $m\left(a, \Gamma_{1}\right)=3$ and $m\left(a, \Gamma_{2}\right)=2$. Thus $\zeta_{2}^{0}(t)=(1-t), \zeta_{2}^{\infty}(t)=1, \zeta_{\left(\Gamma_{1}, \Gamma_{2}\right)}^{0}(t)=(1-t)^{-1}$ and $\zeta_{\left(\Gamma_{1}, \Gamma_{2}\right)}^{\infty}(t)=1$ which coincides with the formulae for $f$ written above.

Example 2. Let $P=x y z+x^{p}+y^{q}+z^{r}$ be a $T_{p, q, r}$ singularity, $\frac{1}{p}+\frac{1}{q}+\frac{1}{r}<1$ and let $Q=x^{d}+y^{d}+z^{d}$ be a homogeneous polynomial of degree $d$. Suppose that $p>q>$ $r>d>3$ and that $p, q$, and $r$ are pairwise prime. Let us compute the zeta-functions of $f=\frac{P}{Q}$ using Theorems 2 and 3 .

(a) It is clear that $f$ is non-degenerated with respect to its Newton pair $\Lambda=\left(\Gamma_{1}, \Gamma_{2}\right)$. Thus

$$
\zeta_{f}^{\bullet}(t)=\zeta_{\Lambda}^{\bullet}(t)=\zeta_{1}^{\bullet}\left(\zeta_{2}^{\bullet}\right)^{-1} \zeta_{3}^{\bullet}(\bullet=0 \text { or } \infty)
$$

One has $\zeta_{1}^{\infty}=\zeta_{2}^{\infty}=1$ and the unique covector which is necessary for computing $\zeta_{3}^{\infty}$ is $a=(1,1,1)$. In this case $m\left(a, \Gamma_{1}\right)=3, m\left(a, \Gamma_{2}\right)=d, \Delta\left(a, \Gamma_{1}\right)=\{(1,1,1)\}$ and $\Delta\left(a, \Gamma_{2}\right)$ is the simplex $\left\{k_{x}+k_{y}+k_{z}=d, k_{x} \geq 0, k_{y} \geq 0, k_{z} \geq 0\right\}$, its two-dimensional volume is equal to $\frac{d^{2}}{2}$. Thus $\zeta_{f}^{\infty}=\left(1-t^{d-3}\right)^{d^{2}}$.

We have

$$
\begin{gathered}
\zeta_{1}^{0}=\left(1-t^{p-d}\right)\left(1-t^{q-d}\right)\left(1-t^{r-d}\right), \\
\zeta_{2}^{0}=\left(1-t^{r(q-d)}\right)\left(1-t^{r(p-d)}\right)\left(1-t^{q(p-d)}\right)\left(1-t^{r-d}\right)^{2 d}\left(1-t^{q-d}\right)^{d} .
\end{gathered}
$$

To compute $\zeta_{3}^{0}$ one has to take into account both covectors $(r q-q-r, r, q),(r, p r-$ $p-r, p)$, and $(q, p, q p-p-q)$, corresponding to two-dimensional faces of $\Gamma_{1}$, and covectors $(1, r-2,1),(r-2,1,1)$, and $(q-2,1,1)$, corresponding to pairs of the form (onedimensional face of $\Gamma_{1}$, one-dimensional face of $\left.\Gamma_{2}\right)$. E.g., for $a=(1, r-2,1), \Delta\left(a, \Gamma_{1}\right)$ (respectively $\left.\Delta\left(a, \Gamma_{2}\right)\right)$ is the segment betweenm $(0,0, r)$ to $(1,1,1)$ (respectively between 
$(d, 0,0)$ and $(0,0, d)$. Pay attention to the "absence of the symmetry": last three covectors are not obtained from each other by permutting the coordinates and the numbers $p, q$, and $r$. This way

$$
\zeta_{3}^{0}=\left(1-t^{r(q-d)}\right)\left(1-t^{r(p-d)}\right)\left(1-t^{q(p-d)}\right)\left(1-t^{r-d}\right)^{2 d}\left(1-t^{q-d}\right)^{d}
$$

and

$$
\zeta_{f}^{0}=\left(1-t^{p-d}\right)\left(1-t^{q-d}\right)\left(1-t^{r-d}\right) .
$$

(b) For computing the zeta-functions of $f$ with the help of Theorem 2, let $\pi:(\mathcal{X}, \mathcal{D}) \rightarrow$ $\left(\mathbb{C}^{3}, 0\right)$ be the blowing-up of the origin in $\mathbb{C}^{3}$ and let $\varphi$ be the lifting $f \circ \pi$ of $f$ to the space $\mathcal{X}$. The exceptional divisor $\mathcal{D}$ is the complex projective plane $\mathbb{C P}^{2}$. Let $H_{1}$ and $H_{2}$ be the strict transforms of the hypersurfaces $\{P=0\}$ and $\{Q=0\}, D_{i}=\mathcal{D} \cap H_{i}$. The curve $D_{1}$ consists of three transversal lines $l_{1}, l_{2}, l_{3}$ and has three singular points $S_{1}=l_{2} \cap l_{3}=(0,0,1), S_{2}=l_{1} \cap l_{3}=(0,1,0)$, and $S_{3}=l_{1} \cap l_{3}=(1,0,0)$. The curve $D_{2}$ is a smooth curve of degree $d$, it intersects $D_{1}$ at $3 d$ different points $\left\{P_{1}, \ldots, P_{3 d}\right\}$.

One has the following natural stratification of the exceptional divisor $\mathcal{D}$ :

(i) 0-dimensional strata $\Lambda_{i}^{0}(i=1,2,3)$, each consisting of one point $S_{i}$;

(ii) 0 -dimensional strata $\Xi_{i}^{0}$ consisting of one point $P_{i}$ each $(i=1, \ldots, 3 d)$;

(iii) 1-dimensional strata $\Xi_{i}^{1}=l_{i} \backslash\left\{D_{2} \cup l_{j} \cup l_{k}\right\}(i=1,2,3)$ and $\Xi_{4}^{1}=D_{2} \backslash D_{1}$;

(iv) 2-dimensional stratum $\Xi^{2}=\mathcal{D} \backslash\left(D_{1} \cup D_{2}\right)$.

It is not difficult to see that $\zeta_{\Xi_{2}}^{0}(t)=1, \zeta_{\Xi_{2}}^{\infty}(t)=1-t^{d-3}$, for each stratum $\Xi$ from $\Xi_{i}^{0}$ $(1 \leq i \leq 3 d), \Xi_{i}^{1}(1 \leq i \leq 4)$ one has $\zeta_{\Xi}^{\bullet}(t)=1(\bullet=0$ or $\infty)$.

In what follows the exceptional divisor $\mathcal{D}$ has the local equation $u=0$. At the point $S_{1}$ the lifting $\varphi$ of the function $f$ is of the form $\frac{u^{3} x_{1} y_{1}+u^{r}+x_{1}^{p} u^{p}+y_{1}^{q} u^{q}}{u^{d} x_{1}^{d}+u^{d} y_{1}^{d}+u^{d}}$. This germ has the same Newton pair as the germ $\frac{u^{3} x_{1} y_{1}+u^{r}}{u^{d}}$. Using theorem 3 one has $\zeta_{\Lambda_{1}^{0}}^{\infty}=1, \zeta_{\Lambda_{1}^{0}}^{\infty}=1-t^{r-d}$. At the point $S_{2}$ the function $\varphi$ has the form $\frac{u^{3} x_{1} z_{1}+z_{1}^{r} u^{r}+x_{1}^{p} u^{p}+u^{q}}{u^{d} x_{1}^{d}+u^{d}+z_{1}^{d} u^{d}}$. It has the same Newton pair as $\frac{u^{3} x_{1} z_{1}+z_{1}^{r} u^{r}+u^{q}}{u^{d}}$. Using Theorem 3 one has $\zeta_{\Lambda_{2}^{0}}^{\infty}(t)=1, \zeta_{\Lambda_{2}^{0}}^{0}(t)=1-t^{q-d}$. Just in the same way $\zeta_{\Lambda_{3}^{0}}^{\infty}(t)=1, \zeta_{\Lambda_{3}^{0}}^{0}(t)=1-t^{p-d}$. Combining these computations together, one has the same results as above (without using a partial resolution).

\section{References}

[1] A'Campo N., La fonction zeta d'une monodromie, Comment. Math. Helv. 50 (1975), $233-248$.

[2] Arnold V.I., Gusein-Zade S.M., Varchenko A.N., Singularities of Differentiable Maps, Vol. II, (Birkhäuser, Boston-Basel- Berlin. 1988). 
[3] Bernshtein D.N., The number of roots of a system of equations, Funct. Anal. Appl. 9 (1975), no. 3, 1-4.

[4] Gusein-Zade S.M., Luengo I., Melle-Hernández A. Partial resolutions and the zeta-function of a singularity, to be pubblish in Comment. Math. Helv.

[5] Khovanski A.G., Newton polyhedra and toroidal varieties, Funct. Anal. Appl. 11 (1977), no. 4, 56-64.

[6] Khovanskir A.G., Newton polyhedra and the genus of complete intersections, Funct. Anal. Appl. 12 (1978), no. 1, 51-61.

[7] Oka M., Principal zeta-function of non-degenerate complete intersection singularity, J. Fac. Sci. Uni. Tokyo Sect. IA, Math. 37 (1990), 11-32.

[8] Varchenko A.N., Zeta function of monodromy and Newton's diagram, Invent. Math., 37 (1976), 253-262.

Addresses:

Sabir M. Gusein-Zade,

Moscow State University,

Faculty of Mathematics and Mechanics.

Moscow, 119899, Russia.

e-mail: sabir@ium.ips.ras.ru

Ignacio Luengo,

Departamento de Algebra,

Facultad de Ciencias Matemáticas,

Universidad Complutense de Madrid.

Ciudad Universitaria, 28040 Madrid, Spain.

e-mail: iluengo@eucmos.sim.ucm.es

Alejandro Melle-Hernández,

Departamento de Geometría y Topología,

Facultad de Ciencias Matemáticas,

Universidad Complutense de Madrid.

Ciudad Universitaria, 28040 Madrid, Spain.

e-mail: amelle@eucmos.sim.ucm.es 